\title{
Neonatal venous cerebral hemorrhage
}

\author{
Report of two cases
}

\author{
Sanjay N. Misra, M.D., ANd Ashish K. Misra, M.D. \\ Department of Neurosurgery, Denver Health Medical Center, University of Colorado Health Sciences \\ Center, Denver, Colorado; and Department of Hematology/Oncology, Children's Hospital at \\ Westmead, Sydney, New South Wales, Australia
}

\begin{abstract}
Intracranial pathological changes can occur as a result of impaired craniocervical venous return. Thrombosis of central venous access catheters was demonstrated in two neonates born at 38 and 27 weeks' gestation. Neither infant developed hemorrhage of prematurity as confirmed on cranial ultrasonography. Clinical evidence of vena cava thrombosis and associated spontaneous intraventricular hemorrhage developed on Day 24 and 36, respectively, and these findings were confirmed on imaging studies. In one infant the hemorrhage was accompanied by communicating hydrocephalus.

The cause of the intracranial disease was attributable to the retrograde cerebral venous congestion. This, together with the primitive venous bed developing in the periventricular region, was associated with the spontaneous hemorrhage in the region of the foramen of Monro.

To the authors' knowledge, this is the first report in the English-language literature of spontaneous neonatal intracerebral hemorrhage, due to thrombosis of the superior or inferior vena cava.

The natural history of this condition is resolution without sequelae after appropriate therapeutic intervention for the vena cava thrombosis.
\end{abstract}

KEY WORDS • cerebral hemorrhage • neonate • venous congestion • thrombosis

Spontaneous intracerebral hemorrhage and secondary communicating hydrocephalus are well described in preterm infants, and result from germinal matrix immaturity compounded by extravascular factors., $, 7,8,14,15,23$ Central venous access catheters are often required in neonates admitted to the neonatal ICU. Venous thrombosis due to the placement of central venous access catheters is a recognized complication..$^{9,16,19,20}$

As a result of long-term venous access, an intraluminal foreign body and altered flow in the vessel are present, both predisposing to thrombosis. The incidence of catheter line-related thrombosis is as high as $28 \%{ }^{9,16,20}$ Infants and neonates may be more prone to this complication because the major venous structures are smaller in diameter than those in adults, and they are also comparatively polycythemic. ${ }^{18}$ The role of cerebral venous obstruction as a cause of various intracranial lesions is well described. ${ }^{5,11,12,21,22}$ Superior vena cava and collateral inferior cava cardiac return are both significant cerebral outflow tracts in normal neonates. ${ }^{17}$ We propose that interference

Abbreviations used in this paper: $\mathrm{CSF}=$ cerebrospinal fluid; ICU = intensive care unit; IVH = intraventricular hemorrhage. with either of these functions by venous thrombosis will result in pathological cerebral venous congestion. Associated with this pathological state is the predisposition for spontaneous intracerebral hemorrhage in neonates beyond the usual period of the hemorrhage of prematurity.

\section{CASE REPORTS}

\section{Case 1}

This female infant was born at 38 weeks' gestation in a normal, uncomplicated delivery. A long femoral access line was placed for the treatment of sepsis. On Day 24 bilateral lower-limb suffusion and edema were noted. In addition, she had become more irritable, with a mild fullness of the anterior fontanelle. Abdominal ultrasonography confirmed inferior vena cava thrombosis. Cranial ultrasonography demonstrated the presence of frontal IVH and slight prominence of the frontal horns. There was evidence on the cervical ultrasonography of superior venous distension but no thrombosis. Mild turbulence of blood flow was also noted in the superior vena cava. The inferior vena cava line was removed, and a subcutaneous dose of low-molecular weight heparin $(1.5 \mathrm{mg} / \mathrm{kg})$ was 
instituted. Repeated cranial ultrasonography at 2 weeks demonstrated clearance of the intracerebral blood and no change in the ventricular status. The circumference growth of the patient's head remained appropriate for age and there were no neurosurgical sequelae.

\section{Case 2}

This female infant with an unexpected birth at 27 weeks' gestation was admitted to the neonatal ICU, requiring ventilation and the placement of an internal jugular line for venous access.

Routine cranial ultrasonography was performed at the time of neonatal ICU admission, and findings were normal (Fig. 1 upper). Because of line-related sepsis, prolonged venous access was required to administer broadspectrum antibiotic agents and provide venous access. Routine cranial ultrasonography on Day 23 revealed no change. On Day 36, facial swelling and suffusion were noted. Chest radiography demonstrated bilateral pleural effusions. Cervical ultrasonography revealed the presence of superior vena cava thrombosis extending from the level of the internal jugular vein to the distal superior vena cava (Fig. 1 center). Cranial ultrasonography demonstrated the presence of IVH and communicating hydrocephalus (Fig. 1 lower).

Venography confirmed the complete occlusion of the superior vena cava (Fig. 2 left). Low-molecular weight heparin therapy was instituted at a dose of $1.5 \mathrm{mg} / \mathrm{kg}$ subcutaneously twice daily. A central venous line was placed at an alternate site and the offending central line was removed. Cranial ultrasonography performed 1 week following treatment demonstrated increased subdural collections and expanded ventricles (Fig. 2 center). There were no further untoward clinical events. The patient's head circumference remained appropriate for her age. She experienced no apneic or bradycardic episodes suggestive of clinically significant intracranial hypertension. Repeated cranial ultrasonography was conducted at 4 weeks after removal of the jugular line and treatment with heparin. This demonstrated clearance of the IVH and decreasing communicating hydrocephalus (Fig. 2 right).

\section{DISCUSSION}

Venous thrombosis resulting from the presence of neonatal central venous access catheters is a recognized complication. The incidence varies (4.5-28\%) depending on the primary vessel involved and is significantly greater for femoral access lines. ${ }^{9,16,20}$ In addition to the presence of an intravascular foreign body, an important predisposing factor is the hematological profile of the developing fetus in the third trimester and in the neonatal period. In the third trimester, there is a progressive increase in hemoglobin. There is a progressive increase in the hemoglobin concentration during the gestational period. By 39 weeks of gestation it has increased to $16.5 \pm 4 \mathrm{~g} / \mathrm{dl} .{ }^{18}$ This relatively high level of hemoglobin is accompanied by a commensurate change in hematological viscosity. In general, neonatal circulating blood has a greater viscosity than that in older patients at a comparable packed cell volume. ${ }^{6}$ In both of our cases, the infants were preterm. On examining the temporal profile of the intracerebral hemorrhage, one

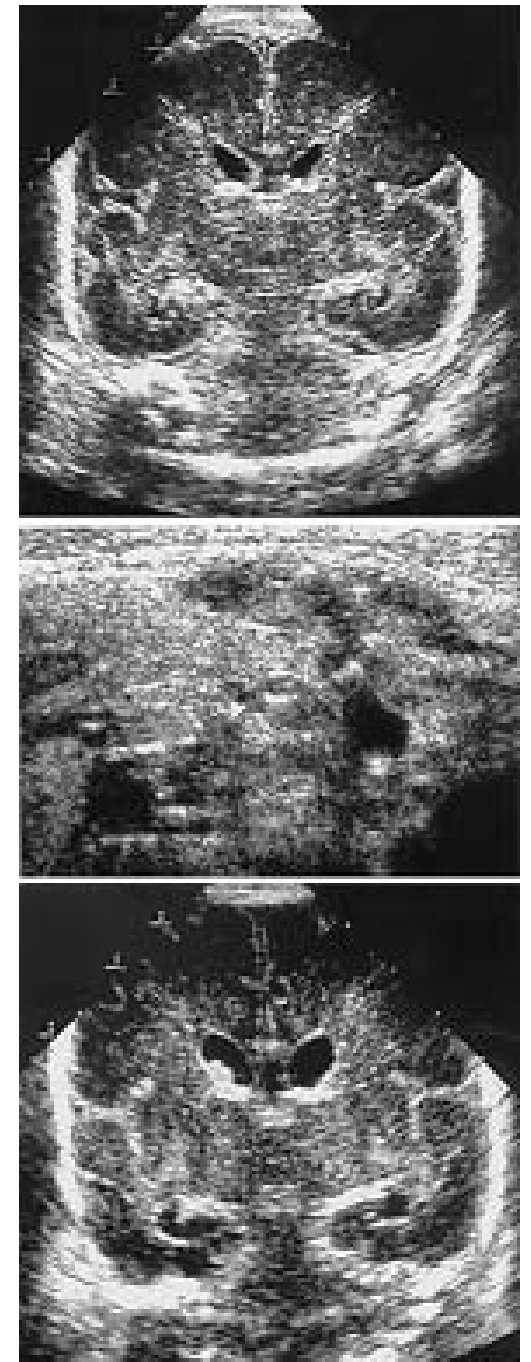

Fig. 1. Case 2. Upper: Cranial ultrasound obtained 24 hours after birth, demonstrating no evidence of intracerebral hemorrhage and normal-appearing ventricles. Center: Cervical ultrasound demonstrating the presence of thrombus surrounding the central line, which completely occludes the superior vena cava (arrow). Center: Cranial ultrasound obtained at time of diagnosis of the superior vena cava occlusion. The IVH is present in the right frontal horn adjacent to the caudate.

can attribute the hemorrhage to the obstruction of craniocerebral venous drainage. The usual form of germinal matrix hemorrhage of prematurity is by the 5 th day of life in $95 \%$ of cases. In each case presented here, the hemorrhage occurred more than 4 weeks after birth and in association with the onset of clinical signs of vena cava thrombosis.

The presence of venous hypertension with retrograde pressure into the cerebral tissue predisposes it to intracranial changes. ${ }^{2,4,5,11,12,21,22}$ The predilection for this in infants is believed to be multifactorial, with important factors being the open cranial sutures and compliance of the neonatal brain and venous system. ${ }^{2,4}$ The development of macrocephaly or neurological instability in infants, accompanying upper- or lower-body congestion, may herald the occurrence on intracerebral hemorrhage with or without communicating hydrocephalus. 

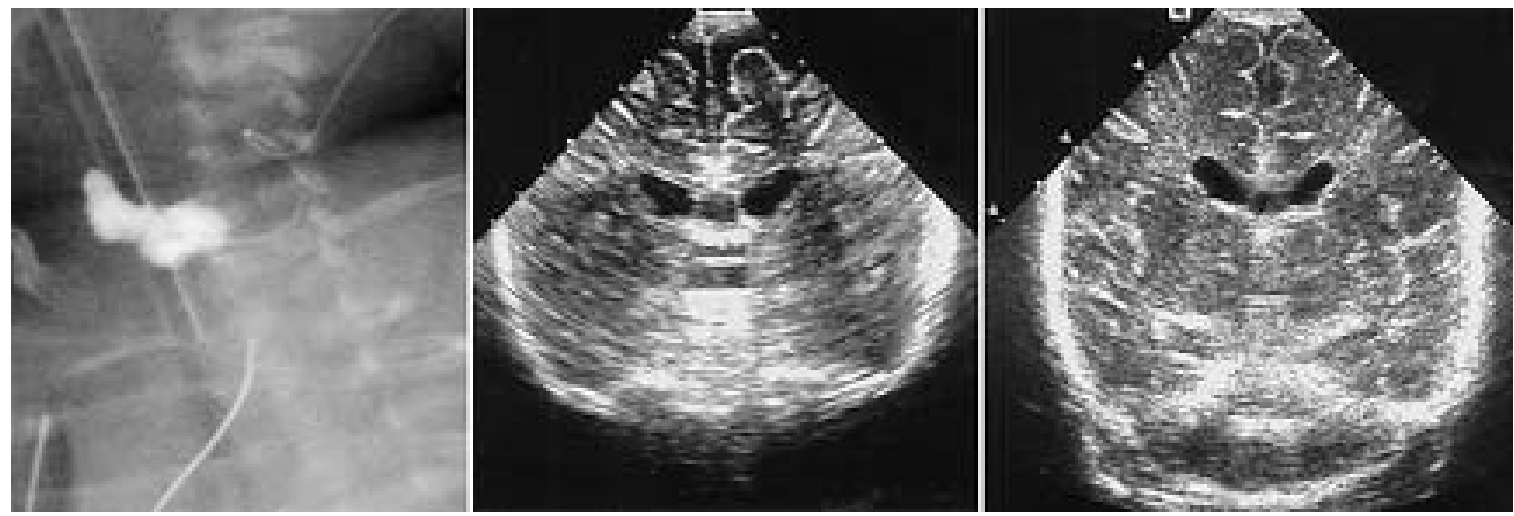

Fig. 2. Case 2. Left: Venogram obtained after instillation of contrast into brachiocephalic vein, demonstrating failure of passage of the contrast into the thorax due to thrombus occupying the superior vena cava. Center: Cranial ultrasound revealing development of bilateral symmetrical extraaxial fluid collections and increase in ventricular size. Frontal IVH has degraded by 1 week posttreatment. Right: Cranial ultrasound confirming decreasing subdural collections after appropriate treatment of the superior vena cava thrombosis 4 weeks posttreatment.

Neonatal cardiovascular investigations have demonstrated that $49 \%$ of the cardiac output in neonates is superior vena cava return. ${ }^{17}$ One can surmise therefore that in neonates with obstructed superior vena cava flow this blood volume would need to be accommodated in other parts of the venous system. The presence of increased volumes in these vessels would then result in decreased flow and relative return pressure via the collateral venous channels.

The distribution of neonatal cerebral and systemic venous return also explains the pathogenesis of the cerebral venous congestion as a result of inferior vena cava thrombosis. There is significant flow of cerebral return via the inferior vena cava with the potential for retrograde venous congestion. ${ }^{10,17}$ Conversely inferior cava thrombosis results in retrograde venous congestion and increases impedance to venous flow.

In studies of retrograde venous cerebral perfusion in canines, investigators have demonstrated intracerebral water content and intracranial pressure elevation as a result of retrograde cerebral perfusion..$^{25}$ In humans, this technique has confirmed the transfer of blood from the cerebral venous side to the arterial side, with passage through the cerebral hemispheres. ${ }^{13}$ Superior vena cava pressure elevation has been associated with an elevation in CSF pressure. ${ }^{24}$ This observation is in keeping with current knowledge that CSF circulation disturbance occurs due to impaired cerebral venous return. ${ }^{5,11,12,21}$ Cardiac return of the craniocerebral perfusate is through the aorta and the azygos, the hemiazygos, and inferior vena cava collaterals. ${ }^{24}$ The observations made in these studies substantiate the proposed pathogenesis for the spontaneous neonatal venous hemorrhage in the two presented cases.

Consideration to the embryology and histology of the germinal matrix in the late fetal period lends additional support to the described neuropathology. The matrix in the region of the caudatothalamic groove is the last region to undergo involution, a process that is underway after 32 to 34 weeks' gestation and completed by term. It is important to note that hemorrhages in the region of the germinal matrix have been demonstrated at the capillary-venule junction and small venules. ${ }^{1}$ The explanation for this may be that these vessels have minimal mesenchymal supporting structures whose development begins to be prominent after 31 to 32 weeks' gestation. These intrinsically weak developing vessels are less able to resist elevated venous pressure. $^{3}$

As demonstrated in these cases, the pathophysiological changes predisposing to intracerebral venous hemorrhage and CSF resorption are corrected by treating the thrombosis. Superior or inferior vena cava thrombosis in neonates results in cerebral venous congestion and responds to the appropriate therapeutic intervention.

\section{CONCLUSIONS}

Interference with central venous return from the head and neck is a predisposing factor for spontaneous neonatal intracerebral hemorrhage. The physiological interference with craniocervical venous return can result from both superior or inferior vena cava obstruction in neonates.

\section{References}

1. Ghazi-Birry HS, Brown WR, Moody DM, et al: Human germinal matrix: venous origin of hemorrhage and vascular characteristics. AJNR 18:219-229, 1997

2. Girard N, Lasjaunias P, Taylor W: Reversible tonsillar prolapse in vein of Galen aneurysmal malformations: report of eight cases and pathophysiological hypothesis. Childs Nerv Syst 10:141-147, 1994

3. Gould SJ, Howard S: An immunohistochemical study of the germinal layer in the late gestation human fetal brain. Neuropathol Appl Neurobiol 13:421-437, 1987

4. Haar FL, Miller CA: Hydrocephalus resulting from superior vena cava thrombosis in an infant. Case report. J Neurosurg 42:597-601, 1975

5. Kollar CD, Johnston IH, Sholler GF: Communicating hydrocephalus secondary to a cardiac tumor compressing the superior vena cava. Childs Nerv Syst 17:117-120, 2001

6. Mackintosh TF, Walker CH: Blood viscosity in the newborn. Arch Dis Child 48:547-553, 1973

7. Mullaart RA, Hopman JC, Rotteveel JJ, et al: Influence of end expiratory pressure on cerebral blood flow in preterm infants. Early Hum Dev 40:157-165, 1995 
8. Mullaart RA, Hopman JC, Rotteveel JJ, et al: Cerebral blood flow velocity and pulsation in neonatal respiratory distress syndrome and periventricular hemorrhage. Pediatr Neurol 16: $118-125,1997$

9. Mulvihill SJ, Fonkalsrud EW: Complications of superior versus inferior vena cava occlusion in infants receiving central total parenteral nutrition. J Pediatr Surg 19:752-757, 1984

10. Oohara K, Usui A, Tanaka M, et al: Determination of organ blood flows during retrograde inferior vena caval perfusion. Ann Thorac Surg 58:139-145, 1994

11. Owler BK, Allan R, Parker G, et al: Pseudotumor cerebri, CSF rhinorrhoea and the role of venous sinus stenting in treatment. Br J Neurosurg 17:79-83, 2003

12. Owler BK, Parker G, Halmagyi GM, et al: Pseudotumor cerebri syndrome: venous sinus obstruction and its treatment with stent placement. J Neurosurg 98:1045-1055, 2003

13. Pagano D, Boivin CM, Faroqui MH, et al: Surgery of the thoracic aorta with hypothermic circulatory arrest: experience with retrograde perfusion via the superior vena cava and demonstration of cerebral perfusion. Eur J Cardiothorac Surg 10: 833-839, 1996

14. Perlman JM, Volpe JJ: Are venous circulatory abnormalities important in the pathogenesis of hemorrhagic and/or ischemic cerebral injury? Pediatrics 80:705-711, 1987

15. Perlman JM, Volpe JJ: Fluctuating blood pressure and intraventricular hemorrhage. Pediatrics 85:620-622, 1990

16. Pippus KG, Giacomantonio JM, Gillis DA, et al: Thrombotic complications of saphenous central venous lines. J Pediatr Surg 29:1218-1219, 1994

17. Salim MA, DiSessa TG, Arheart KL, et al: Contribution of superior vena caval flow to total cardiac output in children. A Doppler echocardiographic study. Circulation 92:1860-1865, 1995
18. Segal GB, Palis J: Hematology of the Newborn, in Beutler E, Lichtman MA, Coller BS, et al (eds): Williams Hematology, ed 6. New York: McGraw-Hill, 2001, pp 77-92

19. Swaniker F, Fonkalsrud EW: Superior and inferior vena caval occlusion in infants receiving total parenteral nutrition. Am Surg 61:877-881, 1995

20. Tanke RB, van Megen R, Daniels O: Thrombus detection on central venous catheters in the neonatal intensive care unit. Angiology 45:477-480, 1994

21. Taylor W: Hydrovenous disorders in pediatric intracranial arteriovenous fistula. Neuroimaging Clin N Am 13:41-53, 2003

22. Taylor WJ, Hayward RD, Lasjaunias P, et al: Enigma of raised intracranial pressure in patients with complex craniosynostosis: the role of abnormal intracranial venous drainage. J Neurosurg 94:377-385, 2001

23. Trommer BL, Groothuis DR, Pasternak JF: Quantitative analysis of cerebral vessels in the newborn puppy: the structure of germinal matrix vessels may predispose to hemorrhage. Pediatr Res 22:23-28, 1987

24. Usui A, Oohara K, Liu TL, et al: Determination of optimum retrograde cerebral perfusion conditions. J Thorac Cardiovasc Surg 107:300-308, 1994

25. Yoshimura N, Okada M, Ota T, et al: Pharmacologic intervention for ischemic brain edema after retrograde cerebral perfusion. J Thorac Cardiovasc Surg 109:1173-1181, 1995

Manuscript received August 25, 2003.

Accepted in final form September 16, 2003.

Address reprint requests to: Sanjay N. Misra, M.D., Department of Neurosurgery, Denver Health Medical Center, University of Colorado Health Sciences Center, 777 Bannock Street, MC 0206, Denver, Colorado 80204. email: Sanjay.Misra@dhha.org. 